\title{
Evaluation of Clinical and Radiological Indicators of Childhood Head Trauma
}

\author{
Çocukluk Çă̆ı Kafa Travmalarının Klinik ve Radyolojik \\ Göstergelerinin Değerlendirilmesi
}

\author{
Münevver Yılmaz®, Ayse Berna Anilø, Murat Anilø, Mehmet Helvacı®
}

Cite as: Yılmaz M, Anil AB, Anil M, Helvacı M. Evaluation of clinical and radiological indicators of childhood head trauma. Forbes J Med. 2021;2(2):79-86.

\section{ABSTRACT}

Objective: The aim of this study is to determine the clinical signs of traumatic brain injury and its long-term effects on prognosis by evaluating the clinical and radiological findings of the patients admitted to the pediatric emergency department due to blunt head trauma.

Method: The cases who applied to the pediatric emergency department due to head trauma were examined prospectively. Glaskow Coma (GCS) and Pediatric Trauma Scores (PTS) were calculated. The patients were evaluated neurologically 6 months after they were discharged.

Results: A total of 707 pediatric patients [mean age: $59.8 \pm 42.6$ months; range: 1 month to 13 years; $263(37.2 \%)$ girls] were evaluated prospectively. Pathology was detected in 101 cases $(45.9 \%)$ [(epidural hematoma, 14; subdural hematoma, 11; brain edema, 36; intracerebral hematoma, 6; subarachnoid hemorrhage, 8; cerebral contusion, 22. Seventy-two (10.1\%) patients had skull fractures.] Seventeen cases $(2.4 \%)$ were operated, and $7(1.4 \%)$ cases were lost. In children aged $<2$ years vomiting, tachypnea, focal neurological findings, multitrauma, GCS $<15$ and low PTS were more common with traumatic brain injury $(p<0.05)$. Vomiting, GCS $<15$ and low PTS were more common in children $>2$ years old and with traumatic brain injury ( $p$ $<0.05)$. Neurological sequelae were not detected in patients aged $<2$ years with mild trauma. Loss of consciousness, pulse rate, respiratory and blood pressure abnormalities, focal neurological findings, low GCS and PTS were more common in children aged $>2$ years and with neurological sequelae $(p<0.05)$.

Conclusion: Physical examination findings, GCS, and PTS levels are useful tools in predicting the short- and long-term consequences of the injury.

Keywords: Pediatric head trauma, Glasgow Coma score, Pediatric Trauma Score, scalp hematoma

öz

Amaç: Bu çalışmanın amacı, künt kafa travması nedeniyle çocuk acil servisine başvuran hastaların yaralanmaya bağlı klinik ve radyolojik bulgularını değerlendirmek; travmatik beyin yaralanmasının klinik işaretlerini ve uzun dönemde prognoza etkilerini belirlemektir.

Yöntem: Çocuk Acil Servisi'ne kafa travması nedeniyle başvuran olgular prospektif olarak incelendi. Olgularda Beyin tomografisi (BT) çekme kararı izleyen hekimin kararına bırakıldı. Olguların Glascow Koma (GKS) ve Pediatrik Travma Skorları (PTS) hesaplandı. Olgular hastaneden taburcu olduktan 6 ay sonra nörolojik olarak değerlendirildi.

Bulgular: Toplam 707 çocuk hasta [ortalama yaș: 59,8 $\pm 42,6$ ay; dağllım: 1 ay-13 yaș; 263 (\%37,2) kız] prospektif olarak değerlendirildi. Yüz bir olguda $(\% 45,9)$ patoloji saptandı [(epidural hematom, 14; subdural hematom ,11; beyin ödemi, 36; intraserebral hematom, 6; subaraknoid kanama, 8; serebral kontüzyon, 22 ve kafa kırı̆̆ı, $72(\% 10,1)]$. On yedi $(2,4 \%)$ hasta operasyona alındı. Yedi $(\% 1,4)$ olgu kaybedildi. İki yaşından küçüklerde kusma, takipne, fokal nörolojik bulgu, multitravma, GKS $<15$ ve düşük PTS saptananlarda travmatik beyin yaralanması daha sıktı $(p<0,05)$. İki yaşından büyüklerde kusma, GKS<15 ve düşük PTS saptananlarda travmatik beyin yaralanması daha sıktı $(p<0,05)$. Altıncı ayda iki yaşından küçük minör tavmalı hastalarda nörolojik sekel saptanmadı. İki yaşından büyüklerde travma sonrası bilinç kaybı öyküsü, nabız sayısı, solunum ve kan basıncı anormallikleri, fokal nörolojik bulgu, düš̈̈k GKS ve PTS nörolojik sekel kalanlarda daha sıktı $(p<0.05)$.

Sonuç: Fizik bakı bulguları, GKS ve PTS düzeyleri yaralanmanın kısa ve uzun dönem sonuçlarını öngörmede faydalı araçlardır.

Anahtar Kelimeler: Pediyatrik kafa travması, Glasgow Koma skoru, Pediatrik Travma Skoru, skalp hematomu

(c) Telif hakkı Forbes Tıp Dergisi. Logos Tıp Yayıncılık tarafindan yayınlanmaktadır.

Bu dergide yayınlanan bütün makaleler Creative Commons 4.0 Uluslararası Lisansı (CC-BY) ile lisanslanmıștır.

(c) Copyright Forbes Journal of Medicine. This journal published by Logos Medical Publishing.

Licenced by Creative Commons Attribution 4.0 International (CC BY)
Received/Geliș: 14.12 .2020

Accepted/Kabul: 26.02.2021

Publication online: 13.08.2021

Sorumlu Yazar/

Corresponding Author: Münevver Yılmaz

Department of Pediatrics, Pamukkale

University Medical Faculty,

Denizli, Turkey

munevveryl@yahoo.com

ORCID: 0000-0002-5076-1479

A.B. Anil 0000-0003-3670-3771

Department of Pediatrics, Izmir Katip Celebi University Medical Faculty, Izmir Turkey

M. Anil 0000-0002-2596-4944 Department of Pediatrics, Izmir Democracy University Medical Faculty, Izmir, Turkey

M. Helvacı 0000-0003-3265-8475

Department of Pediatrics, Health Sciences University, Izmir Tepecik Teaching and Training Hospital, Izmir 


\section{INTRODUCTION}

Traumas are one of the most important causes of child death in the world. ${ }^{1}$ In every 100,000 children, 1,100 children are admitted to the emergency room for head trauma, while 31 of them are hospitalized and 3.4 of them die. ${ }^{2}$ Most common causes of head trauma include falls from heights, and traffic accidents. ${ }^{3}$

Most of the children presented with head trauma are cases with minor head trauma and a small number of children need surgery. ${ }^{3}$ Computed tomography $(\mathrm{CT})$ is an effective and practical method of showing traumatic brain injury in children. However, radiation exposed during CT, can cause cancers in the long term. Therefore, doctors should decide whether or not to perform CT in pediatric patients based on the physical examination findings, age, and cause of the head trauma. $^{3,4}$

The aim of this study was to evaluate the clinical and radiological findings of patients admitted to the pediatric emergency department due to blunt head trauma and to determine the clinical signs of traumatic brain injury and its effects on prognosis in the long term.

\section{MATERIALS AND METHODS}

Between 01.9.2005-31.8.2006, patients under the age of 14 who applied to the Pediatric Emergency Department for head trauma were evaluated prospectively. The patients who were referred to another hospital or refused treatment, and those with missing emergency records were excluded from the study. At admission, Glasgow Coma (GCS) and Pediatric Trauma Scores (PTS) of the cases were calculated. ${ }^{5}$ Those with GCS $13-15$ were evaluated as "minor head trauma". ${ }^{6}$ The scalp hematomas of the cases were divided into four groups according to their size as nonexistent, barely palpable, easily palpable and large scalp hematomas. ${ }^{7}$ In patients aged 24 months or less, the fall distance of 1 meter, and in patients older than 2 years the fall distance of $\geq 1.5$ meters were accepted as the criteria for falling from height. $^{3}$ Falling from a distance less than these heights was considered as simple fall.

The decision to obtain CT was made by the pediatrician who was in duty in the emergency department. No standard algorithm was used. Radiologists evaluated the CTs. The neurosurgeons evaluated the cases for surgical indications. All discharged children were evaluated by telephone calls within the 48th hour, on the 7th day, at the 1st month after trauma. Six months after the trauma, the cases were evaluated neurologically. The cases were divided into $\leq 24$, and $>24$ month- old groups according to their age. The results were analyzed with SPSS 15.0 (SPSS for Windows 15.0, IL, USA). Chi-square, Student-t, One-way ANOVA, Mann-Whitney $U$ and KruskalWallis tests were used. $\mathrm{P}<0.05$ was considered statistically significant.

This study was approved by Tepecik Hospital Education and Scientific Commission as a thesis study.

\section{RESULTS}

A total of 707 pediatric patients [mean age: $59.8 \pm 42.6$ months; median age: 53 months; range: 1 month-13 years; 263 (37.2\%) girls and $444(62.8 \%)$ boys] were evaluated prospectively. When the patients were divided into 2 groups as aged $<2$ (192 patients, 27.2\%) and $>2 \quad(515$ patients $72.8 \%$ ) years, their mean ages were $13.8 \pm 6.3$ and $76.9 \pm 37.3$ months, respectively. The highest number of admissions was in July ( $\mathrm{n}$ : $130 ; 18.4 \%)$. The most common cause of trauma was simple fall (n: 309; 43.7\%) (Table 1).

The median GCS of the patients at admission was 15 points (interquartile range: 12-15). According to the firstly estimated GCS, 665 (94\%) patients had mild, 21 (3\%) had moderate and 21 (3\%) had severe head traumas. The median PTS of the cases was 12 (interquartile range: 7-12). In a total of 100 patients (14.1\%) multiple organ injuries [musculoskeletal injury in 78, (11\%); cervical injury in 10 (1.4\%); abdominal injury in $9(1.3 \%)$; and genitourinary injury in $3(0.04 \%)$ cases ] were detected. 
Table 1. Etiologic distribution of pediatric head traumas by months

\begin{tabular}{|c|c|c|c|c|c|c|c|}
\hline $\begin{array}{l}\text { Months } \\
\text { n (\%) }\end{array}$ & $\begin{array}{c}\text { Simple fall } \\
\text { N: } 309 \\
\text { n (\%) }\end{array}$ & $\begin{array}{c}\text { Falling from a } \\
\text { height } \\
\text { N: } 119 \\
\text { n (\%) }\end{array}$ & $\begin{array}{c}\text { Traffic } \\
\text { accident } \\
\text { N: } 29 \\
\text { n (\%) }\end{array}$ & $\begin{array}{c}\text { Pedestrian } \\
\text { accident } \\
\text { N: } 120 \\
\text { n }(\%)\end{array}$ & $\begin{array}{c}\text { Bicycle } \\
\text { accident } \\
\text { N: } 55 \\
\text { n }(\%)\end{array}$ & $\begin{array}{c}\text { Assault } \\
\text { N: } 7 \\
\text { n (\%) }\end{array}$ & $\begin{array}{l}\text { Other } \\
\text { N: } 68 \\
\text { n (\%) }\end{array}$ \\
\hline January & $\begin{array}{c}11 \\
(42.3)\end{array}$ & $\begin{array}{c}4 \\
(15.4)\end{array}$ & $\begin{array}{c}1 \\
(3.8)\end{array}$ & $\begin{array}{c}3 \\
(11.6)\end{array}$ & 0 & 0 & $\begin{array}{c}7 \\
(26.9)\end{array}$ \\
\hline February & $\begin{array}{c}16 \\
(52.2)\end{array}$ & $\begin{array}{c}2 \\
(6.9)\end{array}$ & $\begin{array}{c}2 \\
(6.9)\end{array}$ & $\begin{array}{c}3 \\
(10.3)\end{array}$ & 0 & $\begin{array}{c}1 \\
(3.4)\end{array}$ & $\begin{array}{c}5 \\
(17.2)\end{array}$ \\
\hline March & $\begin{array}{c}27 \\
(62.8)\end{array}$ & $\begin{array}{c}7 \\
(16.3)\end{array}$ & $\begin{array}{c}1 \\
(2.3)\end{array}$ & $\begin{array}{c}5 \\
(11.6)\end{array}$ & 0 & $\begin{array}{c}2 \\
(2.3)\end{array}$ & $\begin{array}{c}2 \\
(4.7)\end{array}$ \\
\hline April & $\begin{array}{c}12 \\
(25)\end{array}$ & $\begin{array}{c}13 \\
(27.1)\end{array}$ & $\begin{array}{c}5 \\
10.4\end{array}$ & $\begin{array}{c}10 \\
(20.8)\end{array}$ & $\begin{array}{c}3 \\
(6.3)\end{array}$ & $\begin{array}{c}1 \\
(2.1)\end{array}$ & $\begin{array}{c}4 \\
(8.3)\end{array}$ \\
\hline May & $\begin{array}{c}18 \\
(30.5)\end{array}$ & $\begin{array}{c}11 \\
(18.6)\end{array}$ & $\begin{array}{c}3 \\
(5.1)\end{array}$ & $\begin{array}{c}24 \\
(40.7)\end{array}$ & $\begin{array}{c}1 \\
(1.7)\end{array}$ & 0 & $\begin{array}{c}2 \\
(3.4)\end{array}$ \\
\hline June & $\begin{array}{c}34 \\
(31.8)\end{array}$ & $\begin{array}{c}21 \\
(19.6)\end{array}$ & $\begin{array}{c}2 \\
(1.9)\end{array}$ & $\begin{array}{c}30 \\
(28)\end{array}$ & $\begin{array}{c}14 \\
(13.1)\end{array}$ & 0 & $\begin{array}{c}6 \\
(5.6)\end{array}$ \\
\hline July & $\begin{array}{c}67 \\
(51.5)\end{array}$ & $\begin{array}{c}22 \\
(16.9)\end{array}$ & $\begin{array}{c}4 \\
(3.1)\end{array}$ & $\begin{array}{c}11 \\
(8 .)\end{array}$ & $\begin{array}{c}14 \\
(10.8)\end{array}$ & 0 & $\begin{array}{c}12 \\
(9.2)\end{array}$ \\
\hline August & $\begin{array}{c}36 \\
(37.9)\end{array}$ & $\begin{array}{c}15 \\
(15.8)\end{array}$ & $\begin{array}{c}7 \\
(7.4)\end{array}$ & $\begin{array}{c}19 \\
(20)\end{array}$ & $\begin{array}{c}10 \\
(10.5)\end{array}$ & $\begin{array}{c}1 \\
(1.1)\end{array}$ & $\begin{array}{c}7 \\
(7.4)\end{array}$ \\
\hline September & $\begin{array}{c}55 \\
(53.4)\end{array}$ & $\begin{array}{c}19 \\
(18.4)\end{array}$ & $\begin{array}{c}2 \\
(1.9)\end{array}$ & $\begin{array}{c}3 \\
(2.9)\end{array}$ & $\begin{array}{c}13 \\
(12.6)\end{array}$ & $\begin{array}{c}1 \\
(0.9)\end{array}$ & $\begin{array}{c}10 \\
(9.8)\end{array}$ \\
\hline October & $\begin{array}{c}12 \\
(50)\end{array}$ & $\begin{array}{c}3 \\
(12.5)\end{array}$ & $\begin{array}{c}2 \\
(8.3)\end{array}$ & $\begin{array}{c}5 \\
(20.8)\end{array}$ & 0 & 0 & $\begin{array}{c}2 \\
(8.3)\end{array}$ \\
\hline November & $\begin{array}{c}11 \\
(47.6)\end{array}$ & $\begin{array}{c}1 \\
(4.8)\end{array}$ & 0 & $\begin{array}{c}3 \\
(14.2)\end{array}$ & 0 & 0 & $\begin{array}{c}7 \\
(33.3)\end{array}$ \\
\hline December & $\begin{array}{c}11 \\
(50)\end{array}$ & $\begin{array}{c}1 \\
(4.5)\end{array}$ & 0 & $\begin{array}{c}4 \\
(18.2)\end{array}$ & 0 & $\begin{array}{c}2 \\
(9.1)\end{array}$ & $\begin{array}{c}4 \\
(18.2)\end{array}$ \\
\hline
\end{tabular}

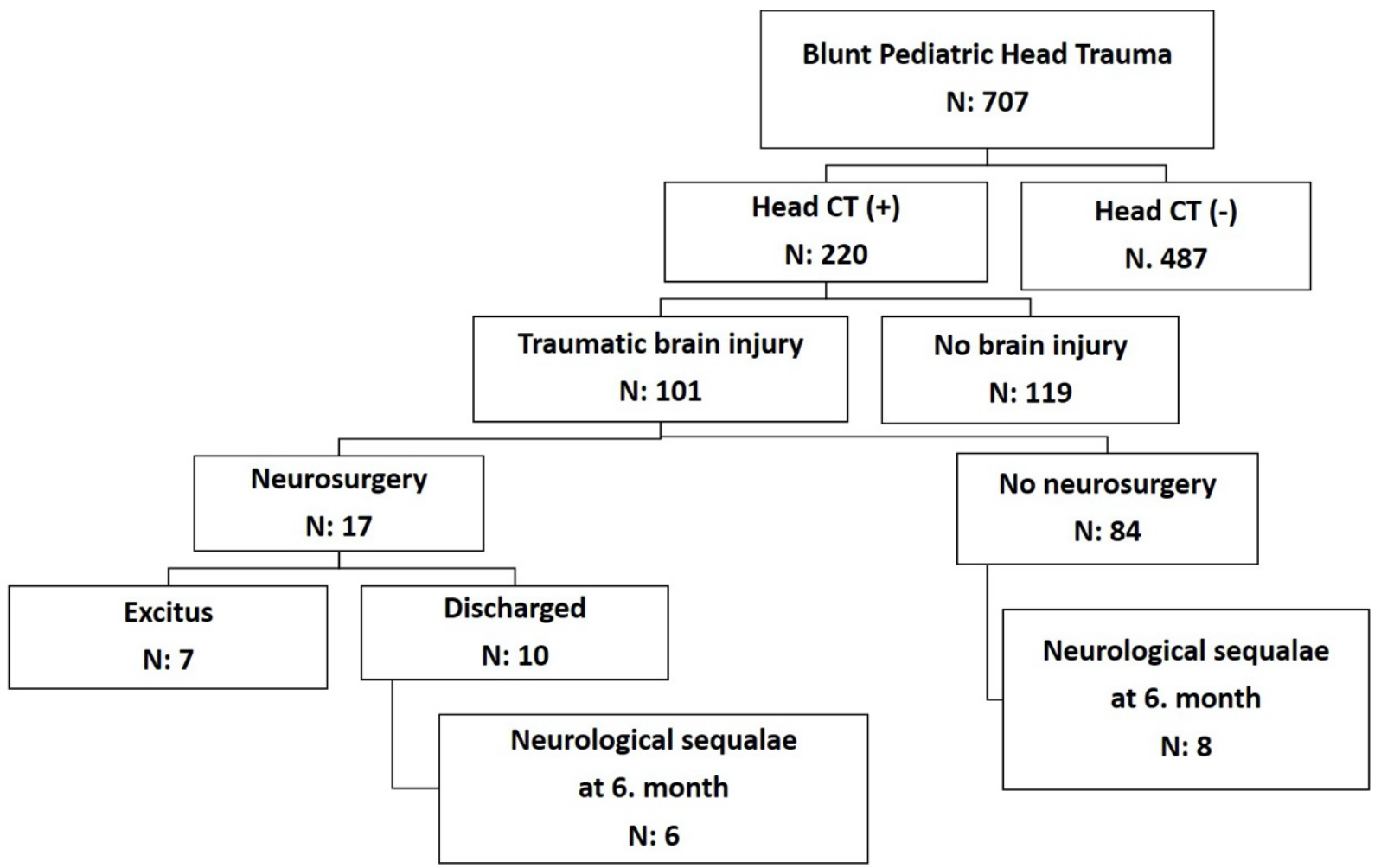

Figure 1. Clinical results of children with blunt head trauma 
A total of 220 patients (31.1\%) underwent CT. Traumatic injury was detected in 101 cases (45.9\% of the cases were identified with CT) [epidural hematoma in $14(1.9 \%)$; subdural hematoma in 11 (1.6\%); brain edema in 36 (5.1\%); intracerebral hematoma $(0.8 \%)$, subarachnoid hemorrhage in $8(1.1 \%)$ and cerebral contusion in 22 (3.1\%) cases]. Seventytwo $(10.1 \%)$ patients had cranial fractures [linear fractures in 63 (8.9\%); depressed fractures in 6 $(0.8 \%)$ and basilar skull fractures in $3(0.4 \%)$ cases]. Hundred and twenty-three (17.4\%) patients were hospitalized. Seventeen (2.4\%) cases were operated. A total of $7(1.4 \%)$ cases exited (Figure 1). When all cases were evaluated at the 6th month after trauma, neurological sequalae were detected in $14(1.7 \%)$ patients including cases with muscle paralysis (n: $8 ; 1 \%$ ), strabismus $(\mathrm{n}: 3 ; 0.4 \%)$, hearing loss $(\mathrm{n}: 1 ; 0.1 \%)$ had, speech impairment (n:1;0.1\%), vision and hearing loss ( $\mathrm{n}: 1 ; 0.1 \%)$.
Sixty-five (33.8\%) out of the 192 patients aged $\leq 2$ years who were admitted to the emergency department due to head trauma had undergone CT. Intracranial injury was detected in 23 (34.5\%) patients (Table 2 ). Among children aged $\leq 2$ years, vomiting, abnormal respiratory pattern (tachypnea, bradypnea, abnormal breathing, apnea, etc.), focal neurological findings and multiple injuries were more common in cases with traumatic brain injury $(\mathrm{p}<0.05)$. Additionally, GCS and PTS were lower in cases with abnormal CT $(p<0.05)$ (Table 2).

When patients with skull fractures were evaluated, only 6 patients with scalp hematoma were found to have fractures. There was no statistically significant relationship between the size of the scalp hematoma and the presence of fracture $(p>$ 0.05). When the relationship between the location of scalp hematoma and the presence of fracture was evaluated, no fractures were detected in any

Table 2. Comparison of the cases with traumatic brain injury or not according to the CT in children $\leq \mathbf{2}$ years old

\begin{tabular}{|c|c|c|c|c|c|c|}
\hline & \multicolumn{3}{|c|}{ All patients } & \multicolumn{3}{|c|}{ Minor head trauma } \\
\hline & $\begin{array}{c}\text { Traumatic brain } \\
\text { injury in CT } \\
n=23\end{array}$ & $\begin{array}{l}\text { Normal CT } \\
n=42\end{array}$ & $\mathbf{p}$ & $\begin{array}{c}\text { Traumatic brain } \\
\text { injury in CT } \\
n=13\end{array}$ & $\begin{array}{c}\text { Normal CT } \\
n=41\end{array}$ & $\mathbf{p}$ \\
\hline Girls, n (\%) & $10(43.5)$ & $22(52.4)$ & 0,670 & $6(46.2)$ & $21(51.2)$ & 0.890 \\
\hline $\begin{array}{l}\text { Mean age, month, } \\
\text { mean } \pm \text { standard deviation }\end{array}$ & $11.5 \pm 7.4$ & $13.2 \pm 5.8$ & 0.800 & $12.0 \pm 7.9$ & $13.0 \pm 5.7$ & 0.780 \\
\hline Seizure, n (\%) & $2(8.2)$ & 5 (11.9) & 0.670 & $2(15.4)$ & $4(9.8)$ & 0.778 \\
\hline Vomiting, n (\%) & $4(17.4)$ & $21(50)$ & $<0.001$ & $3(23.1)$ & $21(51.2)$ & 0.556 \\
\hline Fever, n (\%) & $1(4.3)$ & $1(2.4)$ & 0.800 & 0 & $1(2.4)$ & 0.560 \\
\hline Pulse abnormality, n (\%) & $7(30.4)$ & $5(1.9)$ & 0.344 & 0 & $4(9.8)$ & 0.110 \\
\hline $\begin{array}{l}\text { Respiratory abnormality, n } \\
(\%)\end{array}$ & $4(17.4)$ & $1(2.4)$ & 0.032 & 0 & $1(2.4)$ & 0.890 \\
\hline $\begin{array}{l}\text { Blood pressure abnorma- } \\
\text { lity, n (\%) }\end{array}$ & $3(13)$ & 5 (11.9) & 0.900 & 0 & $4(9.8)$ & 0.552 \\
\hline Scalp hematoma, n (\%) & $10(43.5)$ & $16(38.1)$ & 0.776 & $4(30.8)$ & $16(39)$ & 0.889 \\
\hline $\begin{array}{l}\text { Small scalp hematoma, } \mathrm{n} \\
(\%)\end{array}$ & $2(8.2)$ & $3(7.1)$ & 0,343 & $1(7.7)$ & $3(7.3)$ & 0.900 \\
\hline $\begin{array}{l}\text { Medium scalp hematoma, } \\
\text { n (\%) }\end{array}$ & $3(13)$ & $6(14.3)$ & 0.567 & $1(7.7)$ & $6(14.6)$ & 0.098 \\
\hline $\begin{array}{l}\text { Large scalp hematoma, n } \\
(\%)\end{array}$ & $5(21.7)$ & $7(16.7)$ & 0.455 & $2(15.4)$ & $7(17.1)$ & 0.670 \\
\hline $\begin{array}{l}\text { Focal neurological sign, } n \\
(\%)\end{array}$ & 0 & $2(4.8)$ & $<0.001$ & 0 & $2(14.9)$ & 0.078 \\
\hline Multiple injuries, n (\%) & $5(21.7)$ & $1(2.4)$ & $<0.001$ & $1(7.7)$ & 0 & 0.340 \\
\hline GCS, median (IQR) & $11(8-15)$ & $15(13-15)$ & $<0.001$ & $15(14-15$ & $15(14-15)$ & 0.890 \\
\hline PTS, median (IQR) & $7(3-11)$ & $10(8-12)$ & $<0.001$ & $11(10-12)$ & $10(10-12)$ & 0.894 \\
\hline
\end{tabular}

CT: Computed tomography; GCS: Glasgow Coma Score PTS: Pediatric Trauma Score; IQR: interquartile range 
of the patients with frontal and occipital hematoma, while linear fractures were detected in 2 of the 11 patients with parietal scalp, and in 2 of the 4 patients with temporal scalp hematomas, while basilar fracture were detected in 1 patient $(p<0.001)$.

Hundred and eighty-one out of 192 (94.3\%) patients aged $\leq 2$ years who were admitted to the emergency department due to head trauma had minor head trauma. In this group, 54 (29.8\%) cases had CT. Traumatic injuries (skull fractures in 12 , subdural hematoma in 1 , and cerebral edema in 1 case) were detected in 13 (24.1\%) cases . In this group, no parameters were significant compared to pathologic findings (if any) detected in $\mathrm{CT}(\mathrm{P}>0.05)$. Three (1.7\%) patients with minor head trauma were operated. Neurological sequalae were not detected in any of the cases with minor head trauma (Table 2).
Seven (3.6\%) out of the 192 patients aged $\leq 2$ years old who were admitted to the emergency department due to head trauma were operated. The mean age $(14.1 \pm 6.3$ months vs $8.7 \pm 6.2$ months; $p=0.03$ ), GCS (11 vs $15 ; p<0,001)$ and PTS ( 8 vs $10 ; p<0.001$ ) were comparatively lower in patients aged $\leq 2$ years. Four children $(2.1 \%)$ had neurological sequalae at the end of the 6th month. Focal neurological signs and multiple injuries were more common $(p<0.05)$; GCS and PTS were lower $(p<0.05)$ in cases with neurological sequalae (Table 3 ).

Hundred and fifty-five (29.5\%) out of 515 patients aged $>2$ years who were admitted to the emergency department due to head trauma had undergone CT. Traumatic injury was detected in 77 (49.7\%) patients. Vomiting and focal neurological signs were more common in those with abnormal CT group. GCS and PTS were lower in patients with traumatic brain injury $(p<0.05)$ (Table 4).

Table 3. Comparison of children $\leq 2$ years old in terms of surgical treatment and development of neurological sequelae

\begin{tabular}{|c|c|c|c|c|c|c|}
\hline & $\begin{array}{c}\text { Surgical } \\
\text { treatment }(+) \\
\text { n: } 7\end{array}$ & $\begin{array}{c}\text { Surgical } \\
\text { treatment } \\
(-) \\
\text { n: } 185\end{array}$ & $\mathbf{p}$ & $\begin{array}{c}\text { Neurological } \\
\text { sequelae }(+) \\
n=4\end{array}$ & $\begin{array}{c}\text { Neurological } \\
\text { sequelae (-) } \\
n=188\end{array}$ & $\mathbf{p}$ \\
\hline Girl, n (\%) & $1(14.2)$ & $81(43.8)$ & 0.065 & $1(25)$ & $81(43.1)$ & 0.780 \\
\hline $\begin{array}{l}\text { Mean age, month, } \\
\text { mean } \pm \text { standard deviation }\end{array}$ & $8.7 \pm 6.2$ & $14.1 \pm 6.3$ & 0.030 & $15.2 \pm 4.8$ & $13.8 \pm 6.4$ & 0.087 \\
\hline Seizure, n (\%) & 0 & $7(3.9)$ & 0.654 & 0 & $7(3.7)$ & 0.590 \\
\hline Vomiting, n (\%) & $1(14.2)$ & $30(16.2)$ & 0.890 & 0 & $31(16.5)$ & 0.060 \\
\hline Pulse abnormality, n (\%) & $2(28.6)$ & $19(10.3)$ & 0.344 & $3(75)$ & $18(9.5)$ & 0.051 \\
\hline $\begin{array}{l}\text { Respiratory rate abnormal- } \\
\text { ity, } \mathrm{n}(\%)\end{array}$ & $1(14.2)$ & $5(2.7)$ & 0.760 & $2(50)$ & $4(2.1)$ & 0.055 \\
\hline $\begin{array}{l}\text { Blood pressure abnormali- } \\
\text { ty, } \mathrm{n}(\%)\end{array}$ & $1(14.2)$ & $11(5.9)$ & 0.560 & $2(50)$ & $10(5.3)$ & 0.780 \\
\hline Scalp hematoma, n (\%) & $2(28.6)$ & $54(29)$ & 0.060 & $2(50)$ & $54(28.7)$ & 0.340 \\
\hline $\begin{array}{l}\text { Small scalp hematoma, } \mathrm{n} \\
(\%)\end{array}$ & 0 & $23(12.4)$ & 0.450 & $1(25)$ & $22(11.7)$ & 0.600 \\
\hline $\begin{array}{l}\text { Middle scalp hematoma, } \\
\text { n (\%) }\end{array}$ & $2(28.6)$ & $18(9.7)$ & 0.235 & $1(25)$ & $19(10.1)$ & 0.560 \\
\hline $\begin{array}{l}\text { Large scalp hematoma, } n \\
(\%)\end{array}$ & 0 & $13(7)$ & 0.110 & 0 & $13(6.9)$ & 0.070 \\
\hline $\begin{array}{l}\text { Focal neurological sign, } n \\
\text { (\%) }\end{array}$ & 0 & $37(20)$ & 0.340 & $2(50)$ & $4(2.1)$ & $<0.001$ \\
\hline Multiple injuries, n (\%) & 0 & $6(3.2)$ & 0.980 & $2(50)$ & $11(5.9)$ & $<0.001$ \\
\hline GCS, median (IQR) & $11(9-14)$ & $15(13-15)$ & $<0.001$ & $7(5-9)$ & $15(13-15)$ & $<0.001$ \\
\hline PTS, median (IQR) & $8(5-9)$ & $10(8-12)$ & $<0.001$ & $1(5-9)$ & $10(8-12)$ & $<0.001$ \\
\hline
\end{tabular}

CT: Computed tomography; GCS: Glasgow Coma Score PTS: Pediatric Trauma Score; IQR: interquartile range 
Table 4. Comparison of the cases with head trauma in children $>2$ years old

\begin{tabular}{|c|c|c|c|c|c|c|c|c|c|}
\hline & $\begin{array}{c}\text { Traumatic } \\
\text { brain injury } \\
\text { in } \mathbf{C T} \\
\mathrm{n}=\mathbf{7 8}\end{array}$ & $\begin{array}{c}\text { Normal } \\
\mathbf{C T} \\
\mathbf{n}=\mathbf{7 7}\end{array}$ & $\mathbf{P}$ & $\begin{array}{c}\text { Operation } \\
(+) \\
\text { n: } 10\end{array}$ & $\begin{array}{c}\text { Operation } \\
(-) \\
\text { n: } 505\end{array}$ & $\mathbf{p}$ & $\begin{array}{c}\text { Neurological } \\
\text { sequelae (+) } \\
\text { n: } 10\end{array}$ & $\begin{array}{c}\text { Neurological } \\
\text { sequelae (-) } \\
\text { n: } 505\end{array}$ & $\mathbf{p}$ \\
\hline $\begin{array}{l}\text { Female gender, } \\
\text { n (\%) }\end{array}$ & $32(41)$ & $\begin{array}{c}27 \\
(35.1)\end{array}$ & 0.440 & $3(30)$ & $178(35.2)$ & 0.567 & $3(30)$ & $178(35.2)$ & 0.456 \\
\hline $\begin{array}{l}\text { Loss of } \\
\text { consciousness, } \\
\mathrm{n}(\%)\end{array}$ & $26(33.3)$ & $\begin{array}{c}25 \\
(32.5)\end{array}$ & 0.901 & $5(50)$ & $50(9.9)$ & $<0.001$ & $4(40)$ & $51(10.1)$ & $<0.001$ \\
\hline Seizure, n (\%) & $7(8.9)$ & 7 (10.9) & 0.980 & $1(10)$ & $13(2.6)$ & 0.785 & $1(10)$ & $13(2.6)$ & 0.233 \\
\hline $\begin{array}{l}\text { Vomiting, } \\
\text { n (\%) }\end{array}$ & $32(41)$ & $\begin{array}{c}44 \\
(57.1)\end{array}$ & 0.040 & $3(30)$ & $87(17.2)$ & 0.125 & $2(20)$ & $88(17.4)$ & 0.098 \\
\hline Fever, n (\%) & 0 & $2(2.6)$ & 0.152 & $1(10)$ & $11(2.2)$ & 0.343 & $3(30)$ & $10(1.9)$ & $<0.001$ \\
\hline $\begin{array}{l}\text { Pulse } \\
\text { abnormality, } \\
\text { n (\%) }\end{array}$ & $7(8.9)$ & $2(2.6)$ & 0.095 & $1(10)$ & $29(5.7)$ & 0.451 & $2(20)$ & $27(5.3)$ & $<0.001$ \\
\hline $\begin{array}{l}\text { Respiratory } \\
\text { abnormality, } \\
\text { n (\%) }\end{array}$ & $12(15.4)$ & $8(10.4)$ & 0.356 & $1(10)$ & $31(6.1)$ & 0.123 & $3(30)$ & $29(5.7)$ & $<0.001$ \\
\hline $\begin{array}{l}\text { Blood pressure } \\
\text { abnormality, } \\
\text { n (\%) }\end{array}$ & $9(11.5)$ & $\begin{array}{c}10 \\
(12.9)\end{array}$ & 0.780 & $5(50)$ & 141 (27.9) & 0.065 & $2(20)$ & $144(28.5)$ & 0.065 \\
\hline $\begin{array}{l}\text { Small size } \\
\text { scalp } \\
\text { hematoma, } \\
\text { n (\%) }\end{array}$ & 28 (35.9) & $\begin{array}{c}25 \\
(32.5)\end{array}$ & 0.650 & $2(20)$ & $57(11.3)$ & 0.554 & $0(0)$ & $59(11.7)$ & 0.655 \\
\hline $\begin{array}{l}\text { Medium } \\
\text { size scalp } \\
\text { hematoma, } \\
\text { n (\%) }\end{array}$ & $8(10.2)$ & 7 (10.9) & 0.960 & 0 & $63(12.5)$ & 0.041 & $1(10)$ & $62(12.3)$ & 0.076 \\
\hline $\begin{array}{l}\text { Large size } \\
\text { scalp } \\
\text { hematoma, } \\
\text { n (\%) }\end{array}$ & $9(11.5)$ & $\begin{array}{c}11 \\
(14.3)\end{array}$ & 0.370 & $3(30)$ & 20 (3.9) & 0.022 & $1(10)$ & $22(4.4)$ & 0.230 \\
\hline $\begin{array}{l}\text { Focal } \\
\text { neurological } \\
\text { sign, n (\%) }\end{array}$ & $8(10.2)$ & $2(2.6)$ & 0.041 & $5(50)$ & $50(9.9)$ & $<0.001$ & $4(40)$ & $51(10.1)$ & $<0.001$ \\
\hline $\begin{array}{l}\text { Multiple } \\
\text { traumas, n (\%) }\end{array}$ & $19(24.4)$ & $\begin{array}{c}12 \\
(15.6)\end{array}$ & 0.175 & $1(10)$ & $13(2.6)$ & 0.760 & $1(10)$ & $13(2.6)$ & 0.330 \\
\hline $\begin{array}{l}\text { Age, month, } \\
\text { mean } \pm \\
\text { standard } \\
\text { deviation }\end{array}$ & $74.5 \pm 35.6$ & $\begin{array}{c}73.4 \pm \\
38.8\end{array}$ & 0.721 & $\begin{array}{l}89.7 \pm \\
50.7\end{array}$ & $76.6 \pm 37$ & 0.066 & $74.4 \pm 26.8$ & $76.9 \pm 7.5$ & 0.890 \\
\hline $\begin{array}{l}\text { GCS, median } \\
\text { (IQR) }\end{array}$ & $\begin{array}{c}12 \\
(10-14)\end{array}$ & $\begin{array}{c}14 \\
(11-15)\end{array}$ & $<0.001$ & $\begin{array}{c}11 \\
(10-13)\end{array}$ & $\begin{array}{c}15 \\
(13-15)\end{array}$ & $<0.001$ & $\begin{array}{c}11 \\
(10-14)\end{array}$ & $\begin{array}{c}15 \\
(13-15)\end{array}$ & $<0.001$ \\
\hline $\begin{array}{l}\text { PTS, median } \\
\text { (IQR) }\end{array}$ & $\begin{array}{c}10 \\
(3-9)\end{array}$ & $\begin{array}{c}12 \\
(11-12)\end{array}$ & $<0.001$ & $\begin{array}{c}9 \\
(7-10)\end{array}$ & $\begin{array}{c}11 \\
(10-12)\end{array}$ & $<0.001$ & $\begin{array}{c}4 \\
(6-12)\end{array}$ & $\begin{array}{c}12 \\
(11-12)\end{array}$ & $<0.001$ \\
\hline
\end{tabular}

CT: Computed tomography; GCS: Glasgow Coma Score PTS: Pediatric Trauma Score; IQR: interquartile range

Ten $(1.9 \%)$ cases older than two years were operated. Post-traumatic loss of consciousness $(p<0.001)$, medium and large scalp hematoma $(p=0.04$ and $p=0.02$ ) were more common in children undergoing operation. GCS $(p<0.001)$ and PTS $(p=0.001)$ were lower in patients who needed surgery (Table 4).
Neurological sequalae were detected in 10 (1.9\%) of 515 patients aged $>2$ years after 6 months. Posttraumatic loss of consciousness $(p<0.001)$, abnormal pulse rate $(p<0.001)$, abnormal blood pressure $(p<0.001)$, respiratory abnormality $(p<0.001)$, fever $(p<0.001)$, and existence of focal neurological finding $(p<0.001)$ were more 
common, while GCS $(p<0.001)$ and PTS $(p<0.001)$ were lower.

Four hundred and eighty-two (93.6\%) out of 525 patients aged $>2$ years had mild head trauma. Of these, 123 (25.5\%) underwent CT and 52 (42.3\%) had traumatic brain injury. Only PTS was significantly lower in patients with mild head trauma who had traumatic brain injury $(p=0.01)$. Of these patients, $5(1 \%)$ were operated and 4 $(0.8 \%)$ had neurological sequalae.

\section{DISCUSSION}

In our study, more than 700 pediatric head traumas were evaluated prospectively. Nearly three-quarters of cases were over two years old, and the most frequent hospital admission occurred in July. The most common cause of head trauma was simple falls. Traumatic brain injury was detected in $14.3 \%$ of all pediatric head trauma cases. Of these, $2.4 \%$ were operated and mortality was less than $1 \%$. Neurological sequalae were detected in $1.9 \%$ of survivors. The presence of vomiting, abnormality in vital findings, focal neurological findings, multiple traumas, low GCS and PTS levels, middle / large size scalp hematoma, parietal and/or temporal scalp hematomas were important predictors for clinical outcomes.

The vast majority of head traumas in children is due to falls from a height followed by traffic and bicycle accidents. ${ }^{1,2}$ The results of our study were similar with the literature. In more than half of the cases, the cause of head trauma was fall from a height, followed by traffic and bicycle accidents. Due to the characteristics of the summer season, the pediatric traumas occurred mostly in July.

Traumatic brain injury in $\mathrm{CT}$ is detected between $3-5 \%$ of children under two years of age with minor head traumas. Clinically significant head trauma requires surgery in $1 \%$ and $0.2 \%$ of the cases. ${ }^{3,8}$ The incidence of traumatic brain injury in cases aged $>2$ years with minor head traumas ranges between 3-7\%. Clinically significant head trauma requires surgery in $1 \%$ and $0.1-0.6 \%$ of the cases. ${ }^{3,8,9}$ When GCS decreased, the risk of traumatic brain injury increases.8, ${ }^{8,9}$ In our study, the rates of brain injury and the need of surgery were about twice of the literature. Less than $1 \%$ of the cases deceased. About $2 \%$ of the children had neurological sequalae. The different results obtained may be associated with the characteristics of the countries in which the studies were conducted. Publications in the literature are usually carried out in the developed western countries. The hospital where our study was conducted serves to the lowest socioeconomic region of Izmir, Turkey's third largest city. This may be the reason why traumas are more frequent and more severe.

Change of consciousness in the emergency department, loss of consciousness after trauma, headache and vomiting are common findings in children presenting with head trauma. ${ }^{10,11}$ Clinical signs indicating abnormal level of consciousness such as agitation, lethargy, and GCS $<15$ are the strongest indicators of traumatic brain injury. ${ }^{3}$ One of the most serious pathological consequences of head trauma is increased intracranial pressure. Vomiting, headache, low GCS, focal neurological findings, abnormalities in vital findings are important clinical indicators for increased intracranial pressure. ${ }^{12}$ In our study, vomiting, respiratory abnormality, focal neurological findings, low levels of GCS and PTS were more common in traumatic brain injuries. Similarly, other than vomiting, the same findings were more frequent in patients with neurological sequalae. In other words, clinical signs pointing to pathology in CT also predict long-term prognosis.

Especially in infants, large scalp hematoma, especially hematoma of the temporal or parietal regions is considered one of the important indicators of traumatic brain injury..$^{13}$ In our study, the temporal or parietal scalp hematomas in children $\leq 2$ years old were more often accompanied by cranial fracture. However, no risk factors related to hematoma size were detected in this age group. In those over two years old, the large size of the hematoma was an indicator of a skull fracture. 
In children affected by blunt high-energy trauma, PTS, measured in the emergency department, is a good marker in predicting the need for critical intervention and the risk of mortality. However, considering all traumas, it has a moderate effectiveness in predicting hospitalization. ${ }^{14}$ PTS expresses the level of consciousness, age of the patient, skeletal injury level and airway patency by one number. ${ }^{5}$ In our study, PTS was found as an effective scoring system in predicting traumatic brain injury, the risk for surgery and neurological sequalae in all age groups.

In conclusion, although head trauma is common in children, the risk of related death and neurological sequalae is generally low. Physical examination findings, GCS and PTS levels are useful tools for predicting short- and long-term consequences of pediatric blunt head traumas.

Ethics Committee Approval: This study was approved by Tepecik Hospital Education and Scientific Commission as a thesis study.

Conflict of Interest: None.

\section{Funding: None.}

Informed Consent: Informed consent form was received from the participant parents.

\section{REFERENCES}

1. http://www.cdc.gov/ncipc/wisqars (Accessed on July 14, 2020).

2. Surveillance report of traumatic brain injury-related emergency department visits, hospitaliations, and deaths. Centers for Disease Control and Prevention, 2014. https://www.cdc.gov/traumaticbraininjury/ pdf/TBI-Surveillance-Report-FINAL_508.pdf (accessed April 22, 2020).

3. Kuppermann N, Holmes JF, Dayan PS, et al.: Pediatric Emergency Care Applied Research Network (PECARN). Identification of children at very low risk of clinically-important brain injuries after head trauma: a prospective cohort study. Lancet. 2009;374(9696):1160-70. doi: 10.1016/ S0140-6736(09)61558-0

4. Bressan S, Romanato S, Mion T, Zanconato S, Da Dalt L. Implementation of adapted PECARN decision rule for children with inör head injury in the pediatric emergency department. Acad Emerg Med. 2012;19(7):801-7.

doi: 10.1111/j.1553-2712.2012.01384.x.

5. Furnival RA, Schunk JE. ABCs of scoring systems for pediatric trauma. Pediatr Emerg Care. 1999;15(3):215-23.

doi:10.1097/00006565-199906000-00013

6. Carroll LJ, Cassidy JD, Holm L, Kraus J, Coronado VG; WHO Collaborating Centre Task Force on Mild Traumatic Brain Injury. Methodological issues and research recommendations for mild traumatic brain injury: the WHO Collaborating Centre Task Force on Mild Traumatic Brain Injury. J Rehabil Med. 2004;(43 Suppl):113-25. doi: 10.1080/16501960410023877.

7. Bin SS, Schutzman SA, Greenes DS. Validation of a clinical score to predict skull fracture in head-injured infants. Pediatr Emerg Care. 2010;26(9):633-9. doi: 10.1097/PEC.0b013e3181 ef0440

8. Osmond MH, Klassen TP, Wells GA, et al; Pediatric Emergency Research Canada (PERC) Head Injury Study Group. CATCH: a clinical decision rule minor use of computed tomography in children with minor head injury. CMAJ. 2010;182(4):341-8. doi: 10.1503/cmaj.091421

9. Da Dalt L, Marchi AG, Laudizi L et al. Predictors of intracranial injuries in children after blunt head trauma. Eur J Pediatr. 2006;165(3):142-8.

doi: 10.1007/s00431-005-0019-6

10. Pandor A, Goodacre S, Harnan S, et al. Diagnostic management strategies for adults and children with minor head injury: a systematic review and an economic evaluation. Health Technol Assess. 2011;15(27):1-202.

doi: $10.3310 /$ hta 15270 .

11. Lee LK, Monroe D, Bachman MC, et al. Isolated loss of consciousness in children with minor blunt head trauma. JAMA. Pediatr 2014; 168:837-47. doi: 10.1001/jamapediatrics.2014.361.

12. Miller Ferguson N, Shein SL, Kochanek PM, et al. Intracranial Hypertension and Cerebral Hypoperfusion in Children with Severe Traumatic Brain Injury: Thresholds and Burden in Accidental and Abusive Insults. Pediatr Crit Care Med. 2016;17(5):444-50.

doi: $10.1097 /$ PCC. 0000000000000709 .

13. Schutzman SA, Nigrovic LE, Mannix R. The Infant Scalp Score: A Validated Tool to Stratify Risk of Traumatic Brain Injury in Infants With Isolated Scalp Hematoma. Acad Emerg Med. 2020. doi: 10.1111/acem.14087.

14. Anıl M, Sarıtaş S, Bıcılıoğlu Y, Gökalp G, Kamit Can F, Anıl AB. Pediyatrik Travma Skorunun Çocuk Acil Servisindeki Performansı: Illeriye Yönelik Bir Çalışma. J Pediatr Emerg Intensive Care Med. 2017;4:1-7. doi: $10.4274 /$ cayd. 30074 